\title{
Variation in sleep is associated with diagnosis of late-onset diabetes: a cross-sectional analysis of self-reported data from the first wave of 'Understanding Society' (the UK Household Longitudinal Study)
}

\author{
R.A. Alfawaz, G.R. Law, E.M. Scott and G.TH. Ellison \\ Temporal Influences on Metabolic Events (TIME) Research Group, The Sound Asleep Laboratory, Leeds Institute of \\ Cardiovascular and Metabolic Medicine (LICAMM), School of Medicine, University of Leeds, Leeds LS2 9JT
}

The present study explored the relationship between diabetes and sleep using self-reported data on clinical diagnoses of late-onset (aged $\geqslant 20 \mathrm{yrs}$ ) diabetes and seven components of sleep from the first wave (2009-10) of the nationally representative UK Household Longitudinal Study (UKHLS); $n=29,452$. This involved multinomial logistic regression before and after adjustment for potential confounders, and for body mass index (BMI) and hypertension.

Clinical diagnosis of diabetes (referent: no diagnosis) as predictor of seven sleep components

\begin{tabular}{|c|c|c|c|c|c|c|}
\hline \multirow[b]{2}{*}{ Sleep component (referent) } & \multicolumn{2}{|c|}{ Model $1^{\mathrm{a}}$} & \multicolumn{2}{|c|}{ Model $2^{\mathrm{b}}$} & \multicolumn{2}{|c|}{ Model $3^{\mathrm{c}}$} \\
\hline & RR & $95 \% \mathrm{CI}$ & RR & $95 \% \mathrm{CI}$ & RR & $95 \% \mathrm{CI}$ \\
\hline \multicolumn{7}{|l|}{ Sleep duration $(\geqslant 7 \mathrm{hrs})$} \\
\hline$<5 \mathrm{hrs}$ & $3 \cdot 00$ & $2 \cdot 42-3 \cdot 70$ & 1.71 & $1 \cdot 35-2 \cdot 15$ & $1 \cdot 37$ & $1.08-1.73$ \\
\hline $5 \mathrm{hrs}$ to $<6 \mathrm{hrs}$ & 1.96 & $1 \cdot 64-2 \cdot 35$ & 1.41 & $1 \cdot 17-1.71$ & $1 \cdot 24$ & $1 \cdot 02-1 \cdot 51$ \\
\hline $6 \mathrm{hrs}$ to $<7 \mathrm{hrs}$ & $1 \cdot 33$ & $1 \cdot 17-1 \cdot 52$ & $1 \cdot 13$ & $0 \cdot 99-1 \cdot 30$ & 1.06 & $0.92-1 \cdot 22$ \\
\hline \multicolumn{7}{|c|}{ Sleep latency (cannot get to sleep within $30 \mathrm{~min}$; 'Not during the past month') } \\
\hline 'Less than once a week' & $0 \cdot 82$ & $0 \cdot 70-0 \cdot 96$ & $0 \cdot 94$ & $0 \cdot 80-1 \cdot 10$ & $0 \cdot 94$ & $0 \cdot 80-1 \cdot 11$ \\
\hline Once or twice a week' & $0 \cdot 94$ & $0 \cdot 80-1 \cdot 11$ & 1.04 & $0 \cdot 88-1 \cdot 24$ & 1.01 & $0.84-1.20$ \\
\hline 'Three or more times a week' & 1.29 & $1 \cdot 12-1.49$ & $1 \cdot 23$ & $1.05-1.43$ & 1.09 & $0 \cdot 93-1 \cdot 27$ \\
\hline \multicolumn{7}{|c|}{ Sleep disturbance ('Three or more times a week') } \\
\hline 'Not during the past month' & $0 \cdot 51$ & $0.44-0.60$ & $0 \cdot 80$ & $0.68-0.93$ & $0 \cdot 91$ & $0.77-1.06$ \\
\hline 'Less than once a week' & 0.48 & $0 \cdot 40-0.57$ & $0 \cdot 76$ & $0 \cdot 63-0.91$ & $0 \cdot 86$ & $0.72-1 \cdot 04$ \\
\hline 'Once or twice a week' & 0.57 & $0 \cdot 49-067$ & $0 \cdot 74$ & $0 \cdot 63-0.87$ & $0 \cdot 81$ & $0.68-0.95$ \\
\hline \multicolumn{7}{|c|}{ Cough or snore loudly ('Not during the past month') } \\
\hline 'Less than once a week' & $1 \cdot 28$ & $1 \cdot 07-1.52$ & 1.07 & $0.89-1.28$ & $0 \cdot 97$ & $0 \cdot 81-1 \cdot 17$ \\
\hline Once or twice a week' & $1 \cdot 60$ & $1 \cdot 33-1.94$ & $1 \cdot 15$ & $0.95-1.40$ & $0 \cdot 94$ & $0 \cdot 77-1 \cdot 14$ \\
\hline 'Three or more times a week' & $2 \cdot 69$ & $2 \cdot 36-3 \cdot 07$ & 1.60 & $1 \cdot 39-1 \cdot 84$ & $1 \cdot 18$ & $1 \cdot 02-1 \cdot 37$ \\
\hline \multicolumn{7}{|c|}{ Sleep medication ('Not during the past month') } \\
\hline 'Less than once a week' & 0.57 & $0.40-0.81$ & $0 \cdot 72$ & $0.50-1.03$ & $0 \cdot 75$ & $0.52-1.09$ \\
\hline Once or twice a week' & 1.28 & $0.92-1.78$ & $1 \cdot 17$ & $0.83-1.66$ & $1 \cdot 13$ & $0 \cdot 80-1 \cdot 60$ \\
\hline 'Three or more times a week' & $3 \cdot 28$ & $2 \cdot 85-3 \cdot 77$ & $2 \cdot 01$ & $1 \cdot 73-2 \cdot 34$ & 1.60 & $1.37-1.87$ \\
\hline \multicolumn{7}{|l|}{ Sleep quality ('Fairly good') } \\
\hline 'Very good' & 1.00 & $0 \cdot 87-1 \cdot 15$ & $0 \cdot 85$ & $0.74-0.98$ & $0 \cdot 90$ & $0.78-1.04$ \\
\hline 'Fairly bad' & $1 \cdot 14$ & $0 \cdot 98-1 \cdot 32$ & $1 \cdot 17$ & $1 \cdot 00-1 \cdot 37$ & 1.05 & $0 \cdot 89-1 \cdot 23$ \\
\hline 'Very bad' & $2 \cdot 04$ & $1 \cdot 61-2 \cdot 54$ & 1.40 & $1.09-1.79$ & $1 \cdot 12$ & $0 \cdot 86-1 \cdot 44$ \\
\hline \multicolumn{7}{|c|}{ Trouble staying awake ('Not during the past month') } \\
\hline 'Less than once a week' & 0.74 & $0 \cdot 59-0.91$ & 0.99 & $0 \cdot 79-1 \cdot 24$ & $0 \cdot 95$ & $0 \cdot 76-1 \cdot 19$ \\
\hline Once or twice a week' & $1 \cdot 24$ & $0 \cdot 97-1.59$ & $1 \cdot 34$ & $1.03-1.74$ & $1 \cdot 21$ & $0.92-1.58$ \\
\hline 'Three or more times a week' & $2 \cdot 12$ & $1.53-2.92$ & 1.52 & $1 \cdot 07-2 \cdot 15$ & $1 \cdot 23$ & $0 \cdot 86-1 \cdot 75$ \\
\hline
\end{tabular}

${ }^{\mathrm{a}}$ Unadjusted; ${ }^{\mathrm{b}}$ Adjusted for: sex, age, educational attainment, employment status, household structure ${ }^{(1)}$ and marital status ${ }^{\mathrm{c}}$ Adjusted for: ${ }^{\mathrm{b}}$ and for body mass index and clinical diagnoses of high blood pressur

This is the largest study to-date to demonstrate a strong inverse association between late-onset diabetes and poor sleep, even after adjustment for potential confounding. It is also the first study to demonstrate that this association exists across a range of sleep components. However, because these findings stem from cross-sectional analyses of self-reported data for both the exposure and the outcome, further large-scale studies using longitudinal or experimental designs, and using objective measures of diabetes and/or sleep, are required to exclude the possibility that under-adjustment for latent confounders (particularly those associated with recall or reporting bias) are responsible for the association observed.

1. Fowler H, Ellison GTH, Scott EL, Law GR. (2014) The importance of household composition in epidemiological analyses of sleep: Evidence from the Understanding Society longitudinal panel survey. Open J Epidemiol 4, 45-64. doi:10.4236/ojepi.2014.41009 